\title{
Manufacturing and characterization of targets at IFIN-HH: developing an interdisciplinary body of knowledge
}

\author{
Andreea Mitu ${ }^{1,2, *}$, Nicoleta Florea ${ }^{1}$, Nicolae Mărginean ${ }^{1}$, Raluca Mărginean ${ }^{1}$, and Gheorghe Căta -Danil ${ }^{2}$ \\ ${ }^{1}$ Nuclear Physics Department, "Horia Hulubei” National Institute for Physics and Nuclear Engineering (IFIN-HH), Romania \\ ${ }^{2}$ Physics Department, University POLITEHNICA of Bucharest, Romania
}

\begin{abstract}
Target manufacturing is one fundamental issue in nuclear physics experiments using accelerators. A variety of targets are required, each having to satisfy specific conditions related to the experimental particularities. In this context, a brief description of the target preparation laboratory developed at IFIN-HH is presented in this paper. To fulfill the specific requirements, the laboratory is endowed with high performance equipment for evaporation-condensation (thermal resistance, e-based systems, sputtering) and cold rolling. During the last years, consistent technological improvements were achieved. Target characteristics, quality and reliability are important for our experiments' feasibility in the first place, but also for the degree of confidence in the assumed accuracy. Consequently, XRD, AFM, SEM/EDX and RBS analyses are performed in collaboration with specialized departments from our institute and from other research centers. This paper is meant to synthetize our work so far.
\end{abstract}

\section{Introduction}

The target laboratory in IFIN-HH (National Institute for Physics and Nuclear Engineering - "Horia Hulubei") was developed to respond the experimental requirements with thin films of enriched stable isotopes ranging from tens of nanometers to a few micrometers. It was founded in 2013 [1] in order to fabricate targets mainly for nuclear physics experiments at both the 9 MV Tandem and the $3 \mathrm{MV}$ Tandetron accelerators at IFIN-HH, but also for other research facilities (e.g. CERN, IPN Orsay, LNL). In order to improve the present capabilities of the laboratory, a part of the equipment was upgraded (See Section 1 - Laboratory upgrade).

Moreover, for the manufacturing of reliable targets from materials with extremely high vaporization temperatures in the range of $4500-5500^{\circ} \mathrm{C}$ (e.g. Os, W), the Pulsed Laser Deposition (PLD) technique was developed in collaboration with Photonic Processing of Advanced Materials laboratory (PPAM) from National Institute for Laser, Plasma and Radiation Physics (INFLPR). Also, in the same laboratory, we successfully fulfill thermal decontamination using a hydrogen oven in order to remove the oxygen from the surface of the target, which can give significant interference in the gamma spectra for some types of experiments.

As already mentioned, the final target characteristics strongly affect the precision of data measurements. According to the paper reported by Anna Stolarz, the most important parameters for a completed target are thickness uniformity and impurity identification [2]. In this context, the obtained targets are fully characterized using multiple advanced techniques such as Rutherford Backscattering Spectrometry (RBS), X-ray Diffraction (XRD), Atomic Force Microscopy (AFM), Scanning Electron Microscopy and Energy Dispersive X-ray Spectroscopy (SEM/EDX), which were performed in collaboration with specialized departments from our institute and from other research centers.

In 2016, IFIN-HH has managed to access $€ 2$ million funds through the European Union's "Competitiveness Operational Programme, 2014-2020" for the procurement of high tech equipment to establish the Romania's first national nuclear forensics laboratory. The implementation of the project will provide the technical capabilities for handling and analyzing of different types of materials (radioactive and nonradioactive) including MO (optical microscopy), SEM/EDX and XRD.

Detailed and specific information about all the aforementioned techniques is given and finally the plan for our process and performance in the new configuration is presented in this paper. It appears the manufacturing process combined with the characterization techniques offer a strong perspective for target development methods.

\section{Laboratory upgrade}

The target preparation laboratory was developed to produce self-supported targets or deposited layers on various backings applying high vacuum evaporation or rolling techniques, dedicated to different nuclear physics experiments. As reported [1], the laboratory has two High Vacuum Deposition Systems (TE18-High Vacuum 
Deposition System and Quorum Technologies E6700 Bench Top Evaporator) equipped with thermal evaporation, e-gun and sputtering technologies.

Recently, a second e-beam system produced by Mantis Deposition Systems was integrated in the vacuum deposition chamber of TE18-High Vacuum Deposition System (see Fig. 1). This system has numerous advantages for isotopically enriched film deposition from different materials with thicknesses ranging from a fraction of a monolayer to several hundreds of nanometers. Moreover, according to the studies reported by E.H. Kobisk, J.P. Greene and J.M. Heagney [3-5], it was proven to be very useful for the reduction of oxides having high reduction temperatures $\left(1850-1950^{\circ} \mathrm{C}\right)$ in order to obtain a metallic form of the deposited film. The reduction reaction involves an electron heated crucible and zirconium as reducing agent.

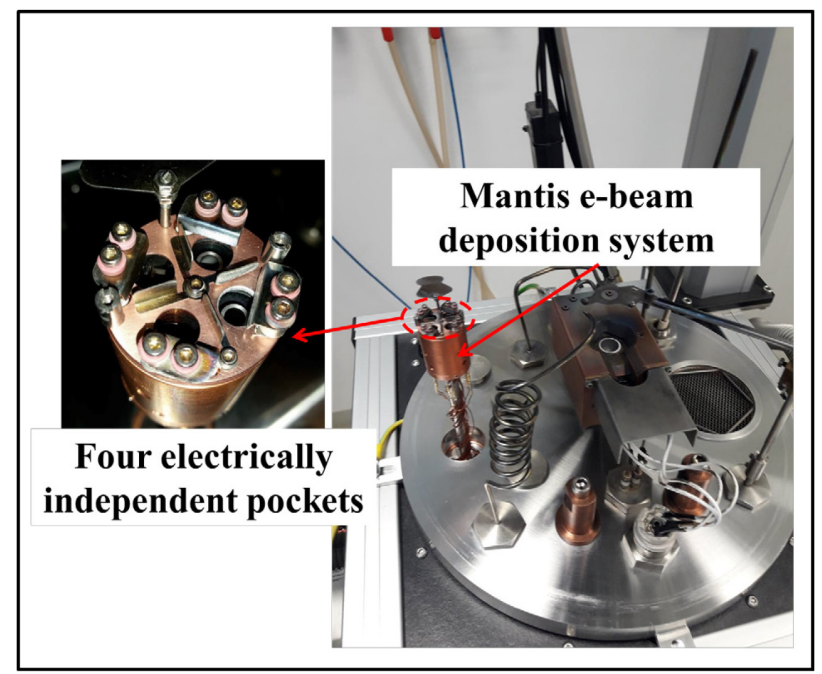

Fig. 1. TE18-High Vacuum Deposition System produced by Intercovamex Company upgraded with e-beam deposition system produced by Mantis Deposition

The Mantis e-beam system is equipped with a QUADEV-C source with a power supply of $500 \mathrm{~W}$ that can be used to heat the target material to temperatures up to $3000^{\circ} \mathrm{C}$. The target rod/crucible is located close to the $\mathrm{W}$ filament. When the current through the filament is sufficient, the electron emission temperature is reached and a stream of electrons is drawn from the filament towards the rod/crucible. Their impact on the rod increases the temperature, which leads to evaporation of the rod or the material placed in the crucible. A QuadEV-C source is used to ensure optimum control of the evaporation rate at low fluxes. This system is equipped with four pockets of $390 \mathrm{~mm}^{3}$; each one uses independent filaments, flux and high voltage channels. It can be used for multilayer deposition by independent monitoring of the deposition rate. The main advantage of this system is that all parameters can be monitored and controlled by means of software applications [6].

At the commissioning date, the target laboratory also included an electrically controlled Durston type DRM 100 rolling mill. The technique of rolling is extremely efficient for self-supported metallic foil production using small amounts of starting material. It allows preparation of metallic foils with areal densities ranging from several hundreds of $\mu \mathrm{g} / \mathrm{cm}^{2}$ to hundreds of $\mathrm{mg} / \mathrm{cm}^{2}$. To increase the quality of the produced metallic foils by rolling, a more powerful, double sided, electrically controlled Durston type FSM 160 VCC rolling mill with variable speed (5-20 rpm) and with touch screen display was acquired (see Fig. 2) [7].

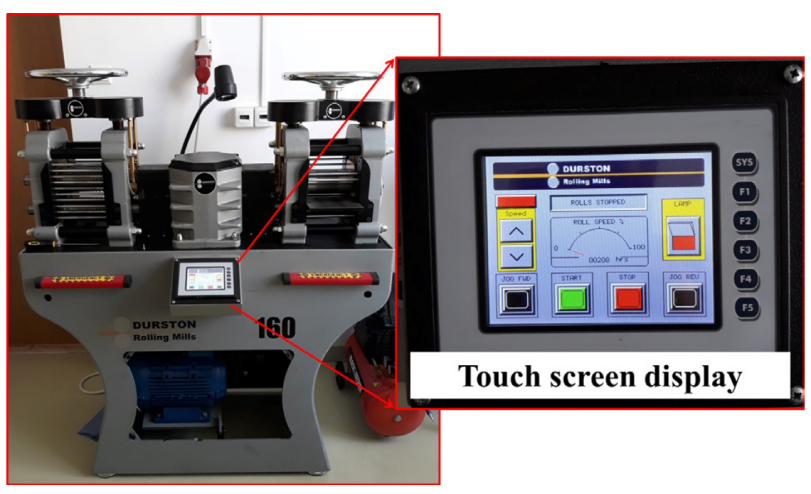

Fig. 2. Durston type FSM 160 VCC power rolling mill, floor standing, double sided with touch screen display

Another important upgrade for our laboratory consists in the procurement of an Atlas $^{\mathrm{TM}}$ Automatic 25 Ton (25T) Hydraulic Press equipped with $40 \mathrm{~mm}$, $32 \mathrm{~mm}, 20 \mathrm{~mm}, 13 \mathrm{~mm}, 10 \mathrm{~mm}$ and $5 \mathrm{~mm}$ evacuable pellet dies, produced by Specac (see Fig. 3) [8]. All the parts of the die are manufactured from hardened stainless steel for optimum quality and durability, and highly polished surfaces for contact with the sample. The applied pressure and release are well controlled. The LCD display shows press status and load conditions providing a digital display of the load applied. The hydraulic press is mainly used for producing selfsupported pellets, which can be further used in experiments as thick targets.

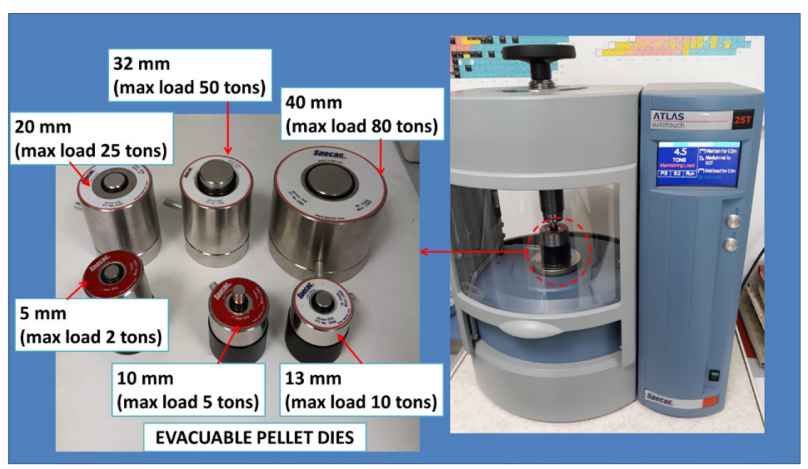

Fig. 3. Atlas ${ }^{\mathrm{TM}}$ Automatic 25Ton (25T) Hydraulic Presses (right) equipped with $40 \mathrm{~mm}, 32 \mathrm{~mm}, 20 \mathrm{~mm}, 13 \mathrm{~mm}, 20 \mathrm{~mm}$ and $5 \mathrm{~mm}$ evacuable pellet dies (left)

The weighing method is very important in the determination of the areal density of foils or deposited targets, combined with the precise surface measuring using a digital optical microscope (Dino-Lite Pro Digital Microscope AM-413TL). Thus, for more stable and accurate weighing results, the new XP56/M analytical balance from Mettler Toledo (Fig. 4) was purchased, offering a weighing capacity of $52 \mathrm{~g}$ with a $0.001 \mathrm{mg}$ readability. 


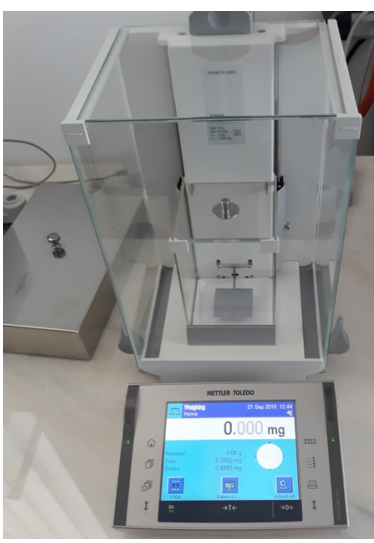

Fig. 4. XP56/M analytical balance

\section{Specific target preparation}

During the last years we prepared a large variety of targets using different methods, either available in the target preparation laboratory, or in collaboration with other laboratories as PPAM from INFLPR, that have complementary equipment (e.g. PLD method). For some specific experiments that required special characteristics, the targets were characterized by means of RBS, AFM, SEM/EDX and XRD techniques. Table 1 shows examples of targets prepared in IFIN-HH.

Table 1: Examples of produced targets by rolling, redox, pulsed lased deposition (PLD) and thermal evaporation (TE)

\begin{tabular}{|c|c|c|c|c|}
\hline Element & Backing & $\begin{array}{c}\text { Areal } \\
\text { density }\end{array}$ & Method & Comment \\
\hline${ }^{64} \mathrm{Ni}$ & $\begin{array}{l}\text { no } \\
\text { backing }\end{array}$ & $\begin{array}{l}1 \mathrm{mg} / \mathrm{cm}^{2} \\
5 \mathrm{mg} / \mathrm{cm}^{2}\end{array}$ & Rolling & a) \\
\hline${ }^{24} \mathrm{Mg}$ & $\begin{array}{l}\text { no } \\
\text { backing }\end{array}$ & $\begin{array}{l}0.2- \\
5 \mathrm{mg} / \mathrm{cm}^{2}\end{array}$ & $\begin{array}{l}\text { Redox/ } \\
\text { rolling }\end{array}$ & b) \\
\hline $\mathrm{W}$ & $\mathrm{Al}$ & $\begin{array}{l}1 \mu \mathrm{m} / \\
20 \mu \mathrm{m}\end{array}$ & PLD & c) \\
\hline Os & $\mathrm{Al}$ & $\begin{array}{l}100 \mathrm{~nm} / \\
20 \mu \mathrm{m} \\
2 \mu \mathrm{m} / \\
20 \mu \mathrm{m}\end{array}$ & PLD & d) \\
\hline${ }^{196} \mathrm{Pt}$ & $\begin{array}{l}\text { no } \\
\text { backing }\end{array}$ & $9 \mathrm{mg} / \mathrm{cm}^{2}$ & rolling & e) \\
\hline${ }^{106} \mathrm{Cd}$ & $\mathrm{Ta}$ & $\begin{array}{l}0.5 \mathrm{mg} / \mathrm{cm}^{2} / \\
2 \mathrm{mg} / \mathrm{cm}^{2}\end{array}$ & rolling & f) \\
\hline $\mathrm{SrF}_{2}$ & $\mathrm{Al}$ & $1.6 \mathrm{mg} / \mathrm{cm}^{2}$ & $\mathrm{TE}$ & g) \\
\hline $\mathrm{SrCO}_{3}$ & $\mathrm{Al}$ & $2.4 \mathrm{mg} / \mathrm{cm}^{2}$ & $\mathrm{TE}$ & h) \\
\hline${ }^{57} \mathrm{Fe}$ & $\begin{array}{l}\text { no } \\
\text { backing }\end{array}$ & $0.4 \mathrm{mg} / \mathrm{cm}^{2}$ & rolling & i) \\
\hline${ }^{96} \mathrm{Zr}$ & $\begin{array}{l}\text { no } \\
\text { backing }\end{array}$ & $\begin{array}{l}1 \mathrm{mg} / \mathrm{cm}^{2} \\
2 \mathrm{mg} / \mathrm{cm}^{2}\end{array}$ & rolling & j) \\
\hline${ }^{65} \mathrm{Cu}$ & $\begin{array}{l}\text { no } \\
\text { backing }\end{array}$ & $7 \mathrm{mg} / \mathrm{cm}^{2}$ & rolling & k) \\
\hline${ }^{64} \mathrm{Ni}$ & $\mathrm{Fe}$ & $\begin{array}{l}0.7 \mathrm{mg} / \mathrm{cm}^{2} / \\
5 \mathrm{mg} / \mathrm{cm}^{2}\end{array}$ & TE & 1) \\
\hline
\end{tabular}

a) $\mathrm{The}{ }^{64} \mathrm{Ni}$ as metallic powder was pressed in a pellet, heated with an e-gun and finally rolled until the desired thickness was obtained;

b) Various ${ }^{24} \mathrm{Mg}$ foils with different thicknesses were prepared starting from ${ }^{24} \mathrm{Mg}$ oxide powder, which was reduced to a metallic form using fresh lanthanum by evaporation-condensation. The material was placed in a Ta boat covered with a copper cooled device;

c) Natural W targets were manufactured by PLD method, $\mathrm{Nd}$ :YAG $1064 \mathrm{~nm}$;

d) Natural Os targets on Al backing were obtained by PLD, Nd:YAG 1064 nm;

e) The powder was sintered in a solid piece using the egun system and the standard rolling procedure was applied;

f) A target of ${ }^{106} \mathrm{Cd}$ deposited on a Ta backing was obtained by rolling both foils together for a plunger type interaction chamber. With a slight pressure between the rolls, the $\mathrm{Cd}$ will sticked to the Ta and could be rolled down together, obtaining a thickness reduced by a factor of four;

g) A homemade cylindrical Ta boat was used for the evaporation process and no difficulties occurred;

h) A similar Ta boat to the previous one (g) was used. Because of the high vaporization temperature of the carbonate an overheating of the copper electrodes can cause contamination of the target;

i) It could be rolled below the limit value estimated in the literature [9], which is $0.5 \mathrm{mg} / \mathrm{cm}^{2}$;

j) The rolling of ${ }^{96} \mathrm{Zr}$ is very difficult. In order to prepare plunger targets with a large surface, special care must be attributed to avoid cracks and holes - a thermal treatment is recommended after every 3 microns thicknesses decrease,

k) ${ }^{65} \mathrm{Cu}$ foils were obtained by rolling. To improve the measurements for the proposed experiment, which was a transfer reaction, a thermal treatment was applied for oxygen decontamination in order to obtain clear $\gamma$-spectra [23];

1) The adhesion between $\mathrm{Ni}$ and $\mathrm{Fe}$ is very poor; to enhance the adhesion between these two materials, the Fe surface used as backing was scratched with a Ted Pella, Pelco Bell Jar Kleen polishing cleaner powder and then washed, thus the surface roughness allowed adhesion of the Ni layer.

\section{Target characterization}

A wide range of nuclear physics experiments requires high-quality and well-characterized targets. In order to fulfill such demands, various techniques were applied. The target thickness is one of the most important parameters, having a considerable contribution in all types of experiments. One of the most common techniques that we use for thickness determination is the $\alpha$-transmission technique using an $\alpha$ source, which contains a mixture of ${ }^{241} \mathrm{Am}$ and ${ }^{244} \mathrm{Cm}$ [1]. Moreover, the thicknesses of different targets were also measured by the RBS technique using $\alpha$ or proton beams delivered by the $3 \mathrm{MV}$ Tandetron accelerator available at IFIN$\mathrm{HH}$. The areal density of the target is very important in all target-beam experiments, as the cross-section uncertainties scale directly with the areal density error. The backscattered protons were registered with an AMETEK type BU-012-050-100 charged particle detector; the RBS spectra were simulated using the 
SIMNRA software package [10-12]. Therefore, in order to investigate the phase composition, the crystalline structure and the chemical purity of the targets, XRD patterns were registered with a PANalytical X'Pert MRD [13], instrument equipped with a fixed divergence slit and fitted with a pixel detector. The X-rays were generated by a $\mathrm{Cu}$ anode polarized at a $40 \mathrm{kV}$ potential, which was bombarded by an electron current of $30 \mathrm{~mA}$. The diffraction data were acquired in a reflection set-up.

AFM analyses were performed with a Park System XE-100 microscope. The studies were conducted in the non-contact mode using silicon cantilevers. The AFM images show topographic information with $256 \times 256$ pixel resolution.

The surface morphology of the targets was investigated by performing SEM/EDX measurements using two types of electron microscopes: the FEI Inspect $\mathrm{S}$ microscope equipped with a $\mathrm{Si}(\mathrm{Li})$ detector EDAX, and the Zeiss EVO MA15 microscope coupled with the Thermo Scientific EDX system.

\section{Specific examples and particularities}

The target preparation laboratory at IFIN-HH in collaboration with INFLPR was involved in the preparation of some particular targets used in different experiments. The target material occasionally exhibits difficulties such as high vaporization temperatures (osmium), high reactivity in air (strontium) or oxygen absorption on the material surface (nickel). Further, a brief description about the preparation and characterization of $\mathrm{Os}, \mathrm{Sr}$ compounds and ${ }^{64} \mathrm{Ni}$ targets will be reported.

\subsection{Osmium targets}

The goal of this research was to prepare Os targets on $\mathrm{Al}$ backings as thin films using the PLD technique. Osmium is the densest stable element; the vaporization temperature is high $\left(\sim 5000^{\circ} \mathrm{C}\right)$, which makes evaporation a challenging process. Two different Os targets with an areal density of 0.2 and $5 \mathrm{mg} / \mathrm{cm}^{2}$ were successfully deposited onto Al backings by the PLD technique using an Nd:YAG laser with a wavelength of $1064 \mathrm{~nm}$ (Fig. 5) [14-17].

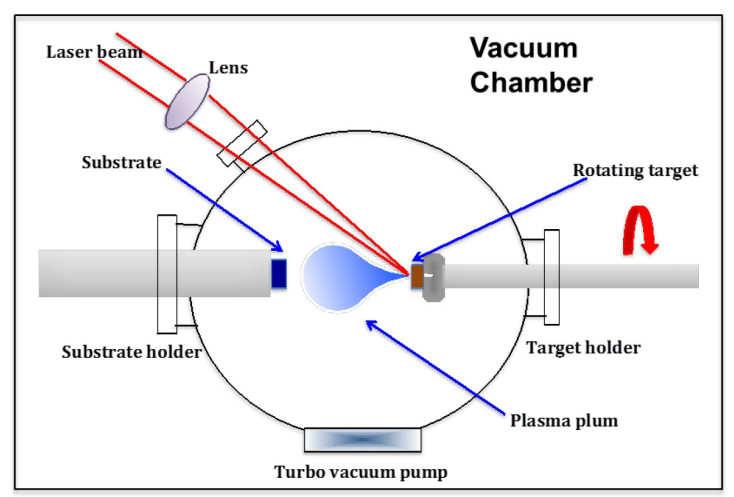

Fig. 5. Experimental set-up for PLD method [17]
Characteristics of the resulting thin films are used in accelerator-based experiments for alpha particle induced reactions. The Os targets were characterized using various techniques as follows. The thicknesses of the Os films were determined by means of RBS, using a $1.8 \mathrm{MeV}$ proton beam. The morphological features of the obtained targets analyzed by AFM and SEM/EDX reveals that the deposited layers $\left(0.2\right.$ and $\left.5 \mathrm{mg} / \mathrm{cm}^{2}\right)$ follow the substrate topography and exhibits good uniformity, meaning it was equally distributed on the whole surface according to the mapping micrographs, while the aluminum backing exhibits imperfections. The EDX and XRD spectra confirm the target purity, as the peaks from the deposited layers are perfectly correlated with the peaks from both the bulk material and the Al backing.

The acquired data during the nuclear activation experiment is presently analyzed in order to establish if all reactions of interest took place. Extended information on this topic and its particularities is available in a research paper [17].

\subsection{Targets from strontium compounds}

Based on previously reported results [18], different $\mathrm{SrF}_{2}$ and $\mathrm{SrCO}_{3}$ targets were prepared by thermal evaporation in similar conditions for nuclear astrophysical experiments with alpha particles accelerated near the Gamow window energy [19, 20]. For this type of experiments, the metallic Sr targets cannot be used, since they exhibit a significant chemical activity and oxidize very fast in air. In this context, the target characteristics (thickness, chemical purity and surface morphology) for both Sr compounds were determined by $\alpha$ transmission measurements, XRD and SEM/EDX analysis, in order to establish which one is better suited for this experiment. The results reveal that $\mathrm{SrF}_{2}$ target exhibits better properties compared to the $\mathrm{SrCO}_{3}$ one. This result was supported by the $\gamma$-ray spectra collected in identical conditions of beam fluency corresponding to the two compounds, showing a clear difference for the intensity of the gamma rays of interest. Partial results of the crosssection measurements are reported in [21]. Detailed information about the strontium compounds target production and characterization, detailed results and discussion can be found in the extended research paper already published [18].

\subsection{Decontamination of ${ }^{64} \mathrm{Ni}$ targets}

According to [22], high purity ${ }^{64} \mathrm{Ni}$ targets were prepared for two neutron transfer reactions of an ${ }^{18} \mathrm{O}$ beam on ${ }^{64} \mathrm{Ni}$ targets at sub-barrier energies [23]. Target preparation considers the $\mathrm{Ni}$ chemical activity in air, as oxygen layers are incorporated and formed on the target surface. This is a major drawback, since gamma rays from ${ }^{16} \mathrm{O}$ target reactions bring a significant contribution to the recorded spectrum. In order to avoid the contamination of the $\gamma$ spectra, a thermal treatment with a hydrogen oven was applied [24]. This way we obtained a significant reduction of the $\mathrm{O}$ contamination (confirmed 
by the gamma lines in the spectra as shown in Fig. 6). Detailed information about the treatment procedure and its effect can be found in our research paper [22].

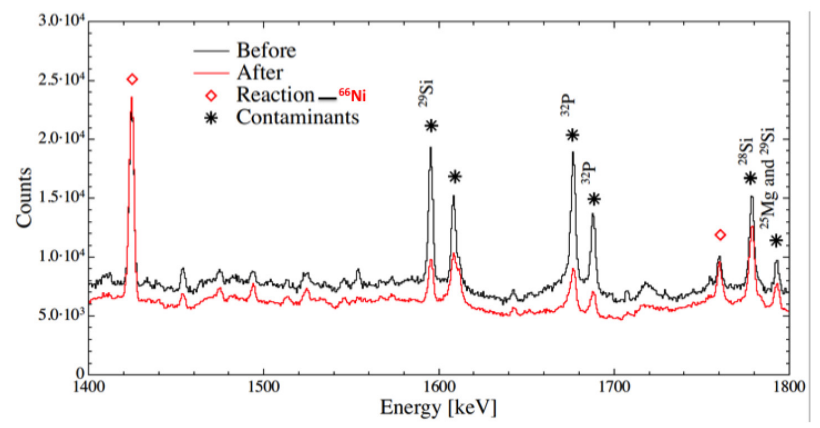

Fig. 6. Gamma spectrum for the reaction of interest before/after thermal treatment of the target [22]

\section{Developing an interdisciplinary collaboration}

IFIN-HH has managed in 2016 to access funds through the European Union's "Competitiveness Operational Programme, 2014-2020" for the procurement of high tech equipment to establish the first Romanian Nuclear Forensics laboratory. The facility will be used for the analysis of forensic materials that contain uranium, plutonium and their descents (see Fig.7). This objective was defined in order to consolidate the national security and research capabilities in forensics.

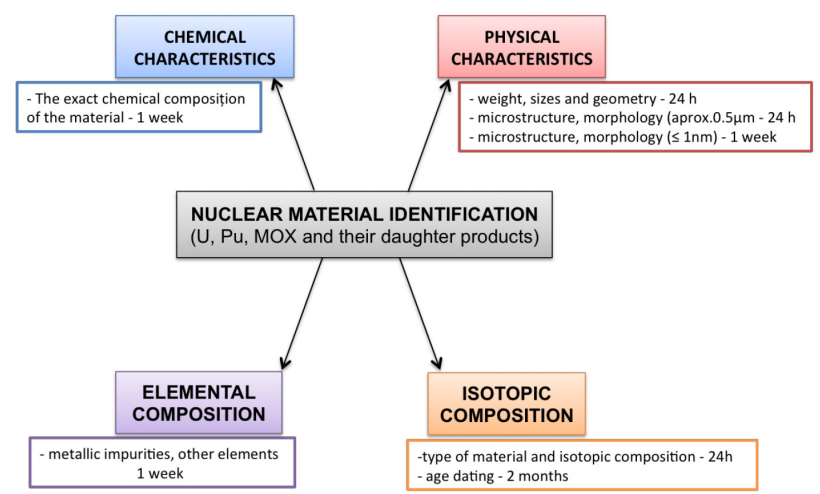

Fig. 7. Overview of analysis techniques required for the categorization and characterization of nuclear materials

Analyses start with physical characteristics: the sample weight is determined with an analytical balance; the sample size and shape are determined by classical methods and a 3D scanner. This information can lead to the first clues on origin (place of extraction or production), processing procedure, conditions for storing or transport, etc.

For the macroscopic parameters, an inverted optical microscope with examination in polarized light capabilities is used. A high-resolution electronic microscope ( $\leq 1 \mathrm{~nm}$ for secondary electrons imaging), equipped with an energy dispersive X-ray detector will be configured in order to allow characterization of a wide range of materials. Then, $\mathrm{XRD}$ is used to characterize phase composition and the crystalline structure of the sample.

Then the chemical composition is considered. The high concentrations of the elements define the material, while minor concentration (impurities) can lead to identification of the material and help determining its origin.

Ion Beam Analysis (IBA) techniques are based on atomic or nuclear interactions of accelerated particle beams (in $\mathrm{MeV}$ energy magnitudes) with atoms or nuclei of an irradiated sample. The Particle Induced X-ray Emission (PIXE) technique is based on the detection of $\mathrm{X}$-rays, which are emitted after the interaction of a beam (in general, relatively low energy protons) with atoms from the sample, allowing qualitative and quantitative analysis. The Particle Induced Gamma-Ray Emission (PIGE) technique is based on the capture of the accelerated particle by the nucleus from the sample, leading to a compound, newly formed nucleus (short life time); followed by emission of protons, alpha particles, and neutrons or/and prompt gamma rays.

Also, Accelerator Mass Spectrometry (AMS) is the most sensitive elemental analysis method available. Isotopic ratios can reach $10^{-16}$ isotope/element; therefore, it is used for measuring low levels of long-lived radionuclides and trace elements.

Gamma-Ray Spectrometry, a non-destructive technique, is used to identify gamma-emitting radioactive nuclides and to determine their concentration.

\section{Conclusion and perspectives}

The target laboratory at IFIN-HH is dedicated to isotopic thin film preparations for various types of nuclear physics experiments with accelerators. The equipment available allows target production by means of thermal evaporation, e-gun, e-beam, sputtering and cold rolling. Additionally, we produce targets by PLD deposition in collaboration with INFLPR. Special care is taken about target quality, which has continuously improved, correlated with our experience and laboratory features. The target characterization in terms of thickness, uniformity, purity, surface morphology and roughness is achieved by means of RBS, SEM/EDX, AFM, and XRD.

With these promising results we have started target production for other international facilities. We are now looking forward to develop a database on thin film characteristics, which would help in refining the aforementioned techniques.

Progress in target characterization is also foreseen along with implementation of the first nuclear forensics laboratory in Romania, which will have modern, high tech equipment, in agreement with ITWG (International Technical Working Group on Nuclear Forensics) recommendations.

To conclude, the manufacturing process combined with characterization techniques lead to innovative target development methods.

This work was supported by the National Institute for Physics and Nuclear Engineering - "Horia Hulubei" (IFIN-HH). 


\section{References}

1. N. M. Florea, L. Stroe, R. Marginean, D. G. Ghiţă, D. Bucurescu, M. Badea, C. Costache, R. Lica, N. Mărginean, C. Mihai, D. V. Moșu, C. R. Niţă, S. Pascu, T. Sava, J Radioanal Nucl Chem, 305, 707-711 (2015)

2. A. Stolarz, J Radioanal Nucl Chem, 299, 913-931 (2014)

3. E.H. Kobisk and H.L. Adair, Nucl. Instrum. Meth. 167, 153 (1979)

4. J. P. Greene and G. E. Thomas, Nucl. Instrum. Methods Phys. Res., Sect. B 61, 575-579 (1991)

5. J.M. Heagney and J.S. Heagney, Proc. World Conf. of the INTDS, A-6850-C, 92 (1977)

6. https://www.mantisdeposition.com/nc/systems

7. http://www.durston.co.uk

8. http://www.specac.com

9. Coating services by MRC, Materials Research GmbH, February (1981)

10. I. Burducea, M. Straticiuc, D.G. Ghiță, D.V. Moşu, C.I. Călinescu, N.C. Podaru, D.J.W. Mous, I. Ursu, N.V. Zamfir, Nucl. Inst. Methods Phys. Res., Sect. B, 359, 12-19, (2015)

11. M. Mayer, SIMNRA User's Guide, Technical Report IPP 9/133, Garching, Germany (1997)

12. https://www.surrey.ac.uk/ati/ibc/files/IBAPitfalls_N ewHandbook.pdf

13. http://www.panalytical.com/XPert3-MRD-XL.htm

14. H.M. Smith and A.F. Tuner, Appl. Opt. 4, 147, (1965)

15. E. Morintale, C. Constantinescu, M. Dinescu, Physics, AUC, 20, 43-56 (2010)

16. D. B. Chrisey and G. K. Hubler, Pulsed Laser Deposition of Thin Films, John Wiley \& Sons Inc., New York (1994)

17. A.Mitu, M. Dumitru, R. Şuvăilă, A. Oprea, I. Gheorghe, P. Mereuță, S. Brajnicov, I. Burducea, N. M. Florea, N. Mărginean, T. Glodariu, M. Dinescu, G. Căta - Danil, Vacuum 161, 162-167 (2019)

18. A. Mitu, A. Oprea, M. Dumitru, N. M. Florea, T. Glodariu, R. Şuvăilă, C. Luculescu, N. Mărginean, M. Dinescu, Gh. Căta-Danil, J Radioanal Nucl Chem, 31, 725 (2018)

19. T. Rauscher, Phys. Rev. C 73, 015804 (2006)

20. T. Rauscher, N. Dauphas, I. Dillmann, C. Fröhlich, Zs. Fülöp, Gy. Gyürky, Rep. Prog. Phys, 76, 066201 (2013)

21. A. Oprea, T. Glodariu, D. Filipescu, I. Gheorghe, A. Mitu, M. Boromiza, D. Bucurescu, C. Costache, I. Cata-Danil, N. Florea, D. G. Ghita, A. Ionescu, N. Mărginean, R. Mărginean, C. Mihai, R. Mihai, A. Negret, C. R. Niţă, A. Olacel, S. Pascu, C. Sotty, R. Şuvăilă, L. Stan, L. Stroe, A. Serban, I. Stiru, S. Toma, A. Turturica, S. Ujeniuc, AEPJ Web of Conferences 146, 01016, (2017)

22. A. Mitu, M. Dunitru, F. Dumitrache, N. Mărginean, R. Şuvăilă, C. R. Niţă, M. Dinescu, Gh. Căta-Danil, U.P.B. Sci. Bull., Series A, 80, Iss. 3 (2018)

23. S. Leoni B. Fornal, N. Mărginean, M. Sferrazza, Y. Tsunoda, T. Otsuka, G. Bocchi, F. C. L. Crespi,
A. Bracco, S. Aydin, M. Boromiza, D. Bucurescu, N. Cieplicka-Orynczak, C. Costache, S. Călinescu, N. Florea, D. G. Ghiţă, T. Glodariu, A. Ionescu, Ł.W. Iskra, M. Krzysiek, R. Mărginean, C. Mihai, R. E. Mihai, A. Mitu, A. Negreţ, C. R. Niţă, A. Olăcel, A. Oprea, S. Pascu, P. Petkov, C. Petrone, G. Porzio, A. Şerban, C. Sotty, L. Stan, I.Ştiru, L. Stroe, R.Şuvăilă, S. Toma, A. Turturică, S. Ujeniuc, C. A. Ur, Phys. Rev. Lett. 118, 162502 (2017)

24. T.A. Utigard, M. Wu, G. Plascencia, T. Marin, Chemical Engineering Science, 60, 2061-2068 (2005) 